\title{
Intención y competencia pedagógica: el uso del aprendizaje colaborativo en la asignatura de matemáticas en secundaria
}

\section{Intention and Pedagogical Competence: Use of Collaborative Learning in the Subject of Mathematics in Secondary School}

\author{
Luis F. Gómez
}

ITESO - Universidad Jesuita de Guadalajara, Jalisco, México.

Recibido: $21-03-16$

Aprobado: 22-05-16

Correspondencia

Email: lgomez@iteso.mx
Citar como:

Gómez, L. (2016). Intención y competencia
pedagógica: el uso del aprendizaje
colaborativo en la asignatura de matemáticas
en secundaria. Propósitos y Representaciones,
4(2). 133-179. Doi: http://dx.doi.
org/10.20511/pyr2016.v4n2.121

(C) Universidad San Ignacio de Loyola, Vicerrectorado de Investigación y Desarrollo, 2016. (e) BY-NC-ND Este artículo se distribuye bajo licencia CC BY-NC-ND 4.0 Internacional (http://creativecommons.org/licenses/by-nc-nd/4.0/). 


\section{Resumen}

En este artículo se analiza la experiencia reportada por profesores de matemáticas de secundarias públicas en Jalisco acerca del trabajo colaborativo de los estudiantes para aprender contenidos y procesos matemáticos. Para ello se revisaron las bitácoras semanales de 30 profesores durante un ciclo escolar y se identificaron las modalidades del trabajo colaborativo, los contenidos o procesos en los que lo utilizaron, los comentarios evaluativos acerca de esta modalidad de trabajo para lograr aprendizajes, así como las decisiones que toman a partir de la reflexión que hacen sobre su eficacia. Se encontró que todos los profesores promovieron el trabajo colaborativo con muy variada frecuencia y en diversas modalidades, principalmente para la resolución de problemas, actividades de descubrimiento, generación de hipótesis, y para extraer información a partir de información dada. Los profesores mostraron comentarios evaluativos diferentes según su experiencia y, a partir de ellos, tomaron la decisión de continuar fomentándolo o de disminuirlo y, por tanto, incrementar la enseñanza directa y el trabajo individual de los alumnos. Se concluye que los profesores intentan promover el aprendizaje mediante la colaboración, pero que carecen de la competencia pedagógica requerida para hacerlo, por lo que no necesariamente tienen los resultados que esperan y algunos regresan a enseñar a través de explicaciones y demostraciones en el pizarrón.

Palabras clave: Aprendizaje colaborativo, enseñanza de las matemáticas, educación secundaria.

\section{Summary}

This review analyzes the experience reported by teachers of mathematics in public junior high schools in Jalisco, México, about the collaborative work of students to learn mathematical content and processes. Weekly logs of 30 teachers were analyzed during one school year to identify modalities of collaborative work, contents or processes taught in this way, evaluative 
comments about this modality of work to achieve learning and the decisions made by teachers after evaluating its efficacy. It was found that all teachers promoted collaborative work but with varied frequency and in different ways, mainly to solve problems, for discovery activities, to generate hypotheses and to extract information from given information. Drawing from their experience, teachers made different evaluations about collaborative learning and consequently they took the decision to continue promoting it or decrease it and in the latter case increase the direct teaching and individual work of students. It is concluded that teachers try to promote learning through collaboration, but lacked the pedagogical competence required to do it, so not all of them had the results they expect, so some of them return to teach through direct teaching, giving explanations and writing demonstrations on the blackboard.

Keywords: Collaborative learning, mathematics education, junior high school. 


\section{Introducción}

En México, las competencias matemáticas de los estudiantes de secundaria evaluados por PISA han sido históricamente bajas. Desde la primera evaluación de PISA (Programa Internacional para la Evaluación de Estudiantes), que se llevó a cabo en el año 2000, México se ubicaba en 387 puntos. En 2003 bajó a 385, en 2006 subió a 406 y, en 2009, a 419. Aunque en las siguientes dos evaluaciones había habido una mejoría, esta fue muy ligera, y en la última evaluación, en 2012, volvió a bajar a 413 puntos. Los estudiantes mexicanos aparecen muy por debajo del promedio de los países miembros de la Organización para la Cooperación y el Desarrollo Económico (OCDE) e incluso de países como Emiratos Árabes Unidos, Kazajistán, Tailandia, Chile y Malasia (OCDE, 2013). Esta baja competencia matemática ha sido atribuida a distintos factores, entre ellos los pedagógicos.

En las décadas recientes, la enseñanza directa de los contenidos escolares por parte de los profesores ha estado muy cuestionada (McDermott, 1993). Se argumenta que esa modalidad de enseñanza no es apropiada para el aprendizaje de todos los contenidos (Marušić \& Sliško, 2014), que los estudiantes aprenden mejor y se interesan más cuando se utilizan métodos interactivos en lugar de las explicaciones del profesor (Andrews, Leonard, Colgrove \& Kalinowski, 2011; Slavish \& Zimbardo, 2012) y que es necesario utilizar distintas modalidades de enseñanza que permitan un mejor aprendizaje, teniendo en cuenta la naturaleza de los contenidos y las características de los alumnos (Brown Wright, 2011).

Una de las modalidades pedagógicas que se ha venido sugiriendo es el aprendizaje colaborativo, que ha mostrado, cuando se implementa adecuadamente, enriquecer el aprendizaje de los alumnos a través de la participación en tareas específicas, al tiempo que promueve las relaciones interpersonales, las habilidades de liderazgo y la autonomía en el aprendizaje (Pai, Sears \& Maeda, 2014). 
En los salones de clases de un grupo de profesores de matemáticas de algunas secundarias públicas de la ciudad de Guadalajara que participaban en un proyecto de mejora de la enseñanza de las matemáticas, el autor de este artículo observó que se utilizaba el aprendizaje colaborativo con diferente frecuencia después de haber tomado un curso sobre aprendizaje colaborativo, por lo que les solicitó que le permitieran revisar sus bitácoras de trabajo para analizar cómo referían el uso de esta modalidad pedagógica en sus aulas.

El objetivo del estudio fue caracterizar el uso de la metodología del aprendizaje colaborativo por parte de un grupo de profesores de matemáticas de secundaria mediante el análisis de las bitácoras que escribieron semanalmente durante un ciclo escolar, con la finalidad de encontrar regularidades que permitieran comprender el uso y evaluación que hacen los docentes de esta modalidad de enseñanza.

\section{El aprendizaje colaborativo.}

El aprendizaje colaborativo es una práctica pedagógica propuesta por John Dewey y Lev Vigotsky desde principios del siglo pasado y que se difundió ampliamente en la década de los 70 a partir de la publicación de informes de investigación sobre los beneficios de este tipo de prácticas (Gillies, 2014). Más tarde, diversos estudios compararon los resultados del aprendizaje cooperativo, competitivo e individual (Johnson, Maruyama, Johnson, Nelson \& Skon, 1981; Johnson \& Johnson, 2002; Hattie, 2009; Slavin, 1996; Gilles, 2008).

Los estudios han mostrado que el trabajo colaborativo promueve el logro académico y la interacción social positiva de los estudiantes en todos los niveles educativos y en una gran variedad de asignaturas (Slavin \& Cooper, 1999; Johnson \& Johnson, 2000). El efecto del trabajo en grupos pequeños ha mostrado tener un impacto en la transferencia del aprendizaje comparado con el aprendizaje individualista. Pai, Sears y Maeda (2014) hicieron un metaanálisis de 124 estudios empíricos que midieron el impacto del aprendizaje en grupos pequeños en la transferencia del aprendizaje y 
encontraron que ese método era superior al del trabajo individual o el competitivo. También las discusiones de grupos pequeños, en la educación en línea, han producido comprensiones más profundas que otras modalidades de trabajo (Bliss \& Lawrence, 2009).

Actualmente, el aprendizaje colaborativo se utiliza y se estudia como medio para el aprendizaje de distintos contenidos en variados escenarios. Por ejemplo: en primaria, para aprender escritura (Guzmán \& Rojas-Drunmond, 2012), para la comprensión de conceptos geográficos (Celikten, Ipekcioglu, Ertepinar \& Geban, 2012) y para promover la comprensión y fluidez lectora (Valdebenito \& Duran, 2013). También se utiliza para el aprendizaje de la vida cotidiana (Mejía-Arauz, Keyser \& Correa, 2013), en ambientes virtuales en inglés como segunda lengua (You, 2014), en educación física y, también, para el aprendizaje de las matemáticas (Hooker, 2011).

Desde 1989, el Consejo Nacional de Profesores de Matemáticas de Estados Unidos, (National Council of Teachers of Mathematics) ha abogado por que se utilice el aprendizaje colaborativo para enseñar esta disciplina debido a que los grupos pequeños proveen un foro en el que los estudiantes preguntan, discuten ideas, cometen errores, aprenden a escuchar las ideas de los otros, ofrecen crítica constructiva y resumen por escrito sus aprendizajes (NCTM, 1989, p. 79). La misma organización incluyó, en uno de sus cinco estándares, la comunicación (NCTM, 2000), la misma que se desarrolla mediante el aprendizaje colaborativo.

El fundamento del trabajo colaborativo se puede encontrar en una de las premisas de la teoría del aprendizaje sociocultural de Vygotsky (1979), que considera que todo proceso psicológico aparece dos veces: primero en el plano interpersonal y, posteriormente, en el plano intrapersonal. Autores que han seguido esta línea de pensamiento señalan que, durante la participación en una actividad, los estudiantes construyen nuevas comprensiones y conocimientos (Mercer \& Littleton, 2007; Rogoff, 2003). Las formas de acción compartidas se internalizan a través de dispositivos semióticos y pasan a formar parte del funcionamiento intrapersonal del individuo (Wertsch, 
1988). Lo que se ejecuta en el plano intrapersonal se construye gradualmente en el plano interpersonal.

La participación en la actividad hace que el estudiante aprenda mediante mecanismos semióticos, pero no basta ponerlos juntos para que aprendan, sino que se requiere diseñar la estructura de la actividad para que ocurra el proceso intencionado y enseñar a los alumnos a participar, pues en ocasiones no saben cómo proceder, cómo incorporar las aportaciones discordantes ni qué hacer con quienes aportan poco (Gillies, 2014). Tomando las ideas de Johnson y Johnson (1990), Gillies (2014) desarrolló una estructura con cinco elementos para evitar estas dificultades: el primero de ellos es establecer un estado de interdependencia positiva para lograr la meta, en la que los alumnos entiendan que no basta con hacer su parte, sino que todos contribuyan; el segundo elemento es que se pide cuenta de manera individual a cada estudiante sobre su aportación; el tercero es el desarrollo de habilidades para manejar la conducta e interacciones de manera que lleven a la meta; el cuarto es la promoción de la interacción, y el último es el procesamiento grupal que consiste en que los miembros del grupo reflexionen acerca de su desempeño, reconozcan lo positivo e identifiquen lo que necesitan para lograr la meta del equipo.

Millis (2014), a partir de una amplia revisión de la literatura, señala siete principios del trabajo colaborativo que promueven el aprendizaje de los alumnos. Algunos de ellos son similares a los elementos de Gillies: 1) una estructura definida, 2) poner en el centro la resolución de problemas, 3) formar grupos heterogéneos, 4) utilizar estructuras cooperativas que promuevan el aprendizaje profundo, 5) establecer la interdependencia positiva pero con la responsabilidad individual, 6) reflexionar sobre el proceso de trabajo (procesamiento grupal) y 7) el desarrollo de habilidades sociales y de liderazgo.

Moliner, Flores y Duran (2011) han profundizado en una de las modalidades del trabajo colaborativo: la tutoría entre iguales, en donde un estudiante asume de manera fija el papel de tutor de otro menos avanzado, o 
donde hay un intercambio de roles y ambos fungen como tutor y tutorado en distinto momento.

Para este trabajo, siguiendo la definición de Johnson, Johnson y Holubec (1999), se entenderá el aprendizaje colaborativo como "el empleo didáctico de grupos reducidos en los que los alumnos trabajan juntos para maximizar su propio aprendizaje y el de los demás" (p. 14).

\section{Método}

La investigación fue de tipo cualitativo, dado que interesaba caracterizar el uso de la metodología del aprendizaje cooperativo por parte de profesores de matemáticas de secundaria. El interés del estudio se centró en la experiencia reportada por los docentes a través de informes escritos. Las preguntas que guiaron la investigación fueron: ¿Cómo informan los profesores que implementan el trabajo colaborativo en las aulas? ¿Cómo describen el proceso de trabajo? ¿Para qué tipo de contenidos o procesos utilizan esta modalidad de actividad de aprendizaje? ¿Qué ventajas y dificultades encuentran? ¿Cuál es la valoración que hacen del trabajo? ¿Qué decisiones toman a partir de las valoraciones? ¿Cómo se compara el trabajo que realizaron estos profesores con lo que señala la teoría como aprendizaje colaborativo?

El grupo participante fue de 30 docentes que enseñan matemáticas en primer grado de secundaria en escuelas públicas de la ciudad de Guadalajara. Fueron $53.3 \%$ de mujeres y $46.6 \%$ de hombres, quienes escribieron en

promedio 27 entradas en sus bitácoras semanales durante el ciclo escolar 2012-2013, como puede verse en la Tabla 1.

\section{Tabla 1.}

Participantes y corpus de datos.

\begin{tabular}{lccc}
\hline Participantes & Número & $\mathbf{N}^{\circ}$ de entradas & Porcentaje de entregas \\
\hline Mujeres & 16 & 358 & $90.4 \%$ \\
Hombres & 14 & 453 & $92.9 \%$ \\
\hline
\end{tabular}


Los profesores eran licenciados en educación matemática e ingenieros. Los primeros tienen conocimiento pedagógico, pero menos conocimiento de la disciplina que los ingenieros. Estos últimos tienen conocimiento de las matemáticas y de su aplicación, pero no tienen el conocimiento de aspectos pedagógicos; sin embargo, reciben capacitación sobre este tema por parte de la Secretaría de Educación.

Se invitó a los docentes a participar en un proyecto de investigación que les ofreció capacitación sobre la enseñanza de las matemáticas y acompañamiento durante un periodo escolar a través de una comunidad virtual de práctica. Por su parte, ellos tenían que participar semanalmente en un foro virtual y subir, con esa misma periodicidad, una bitácora de sus actividades y reflexiones. Parte de los resultados ya ha sido publicada (Gómez \& Silas, 2012; Gómez, Silas \& Miranda, 2015; Gómez \& Silas, 2016). En este artículo se analiza únicamente el aprendizaje colaborativo reportado por los profesores en sus bitácoras.

El corpus de datos estuvo constituido por 30 bitácoras -diarios del profesor- con 811 entradas en total; es decir, cada bitácora fue actualizada, en promedio, 27 veces. En ellas, los docentes registraron semanalmente, durante el periodo escolar 2012-2013, lo que consideraban relevante de su práctica, ya sea en la fase de planeación, implementación o reflexión posterior. El formato era libre y la extensión variable (entre 3 y 8 páginas), por lo que algunos enfatizaban la planeación, otros el trabajo en el aula y algunos más su reflexión sobre el proceso, pero todas contenían esos tres elementos. Dado que no había obligatoriedad, solo un acuerdo, hubo semanas en que algunos de ellos no escribieron. Los docentes se leían y comentaban sobre el trabajo de los otros (Gómez \& Silas, 2016). Uno de ellos refirió que, debido a la virtualidad, no le preocupaba exponer sus limitaciones.

La utilización periódica del diario del profesor "permite reflejar el punto de vista del autor sobre los procesos más significativos de la dinámica en la que está inmerso" (Porlán y Martín, 1991, p. 23). El trabajo se centró en la percepción subjetiva que los docentes tienen de su práctica y de las 
decisiones que toman, por lo que lo subjetivo es objeto de investigación (Zabalza, 2004).

El proceso de análisis consistió en reducir la información eligiendo como unidad temática el trabajo colaborativo; luego se identificaron y clasificaron los elementos: modalidades de trabajo colaborativo, comentarios evaluativos de los profesores, ejemplos de prácticas, situaciones propicias para este tipo de actividad y decisiones tomadas por los docentes, lo que Straus (1987) llama codificación abierta. Posteriormente, la información se desplegó en matrices (Miles \& Huberman, 1994) para analizarla y extraer conclusiones mediante un proceso de inferencia inductiva, y a partir de ello se caracterizó el trabajo colaborativo que se lleva a cabo en este conjunto de aulas de secundaria, desde la percepción de los profesores autores de las bitácoras. Finalmente se comparó su trabajo con lo que en la teoría se denomina aprendizaje colaborativo.

En el análisis, para hacer referencia a las bitácoras citadas se anotan las iniciales del profesor y el número de página en donde se encuentra la información.

\section{Resultados}

El análisis de las bitácoras muestra que en 473 ocasiones se hizo mención al trabajo colaborativo en el aula, con variaciones muy amplias entre unos profesores y otros, desde 35 ocasiones quien más utilizó esta modalidad de trabajo, hasta 3 quien menos lo hizo. Enseguida se muestra cómo fue ese trabajo colaborativo, qué contenidos se utilizaron, cómo fue la valoración de los docentes y qué decisiones tomaron a partir de esta.

\section{EI proceso y las modalidades del trabajo colaborativo.}

El trabajo colaborativo que se reporta estuvo organizado de diversas maneras: equipos pequeños (346 veces), trabajo en binas (91 veces) y trabajo con tutores (36 veces). Los profesores que muestran un trabajo más organizado 
preferían que los alumnos trabajaran en binas para asegurar su participación continua, aunque en ocasiones permitían agrupaciones de tres integrantes. Otros formaban equipos de cuatro o más estudiantes, pero en algunos casos reportaban desorden y falta de trabajo en los equipos. La mención más recurrente fue que los equipos eran de cuatro alumnos. Por lo general, los docentes asignaban a los estudiantes a los grupos, pero algunos de ellos permitían que se unieran por afinidad o por contigüidad de asientos.

Algunos profesores expresaron sus razones para el tipo de grupo colaborativo que formaban; por ejemplo: "En binas cuando se requiere un poco de apoyo de uno a otro" (VT, p. 15), para mantener el orden, cuando el trabajo es sencillo o cuando la actividad es de ejercitación y repaso.

Algunos también refieren que los organizan en grupos cuando la actividad exige la participación de varios alumnos que hagan diversas partes del trabajo grupal, pero que "no pasen de tres o cuatro integrantes" para que todos trabajen. También cuando las actividades son muy complejas o de descubrimiento, en las cuales se requiere que sean varias personas que aporten sus puntos de vista al equipo. Sin embargo, la mayoría de los docentes no dan razones para el tipo de grupalidad que eligen.

Aunque hay profesores que tienen una preferencia por el trabajo en binas y otros por la formación de equipos de cuatro miembros, en ocasiones el tamaño del equipo dependió de la disponibilidad de materiales para una actividad determinada.

El trabajo con tutores consistió en que un alumno con más conocimiento se encargaba de animar y asesorar el trabajo de un grupo pequeño de sus compañeros. Esta modalidad de trabajo se utilizó, de acuerdo con los profesores, debido a que "no es lo mismo mis palabras a las palabras de sus compañeros ni su explicación" (SN, p. 18). El docente considera que un alumno que ya comprendió puede explicar a sus compañeros en una manera y con un lenguaje que no posee el profesor; además, considera que las experiencias compartidas que tienen los estudiantes permiten que 
el "monitor" las utilice para lograr que su compañero comprenda el tema matemático que se aborda.

Aunque en ese trabajo se creaban parejas de alumnos que asumían una relación asimétrica, pues uno fungía como tutor y otro como tutorado, que trabajaban para conseguir un mismo objetivo, hacía falta un elemento clave que señala Duran (2006): la interacción regulada por el docente.

El proceso de trabajo tenía, invariablemente, la siguiente estructura, sin importar que fuera trabajo en binas, grupo pequeños o monitoreo: planteamiento de un problema o una actividad de descubrimiento, trabajo colaborativo por parte de los alumnos, observación y retroalimentación del trabajo por parte del profesor a algunos de los equipos, puesta en común de los resultados de todos o algunos de los equipos. En esta última fase, el docente y los alumnos decían si las soluciones eran correctas o no y las razones para su juicio.

Tanto en los grupos pequeños como en las binas hubo tres maneras en que los alumnos fueron agrupados: por asignación del profesor, por preferencia del alumno o por contigüidad. Algunos docentes utilizan una sola de estas maneras de agrupar, mientras que otros las utilizan todas, dependiendo de la situación, como puede verse en lo que escribe uno de los profesores: No existe una organización fija; algunas veces, algunos alumnos me piden sentarse junto a mi en el escritorio y trabajan mejor alli; otras veces los pongo en binas, otras en equipos; si están muy inquietos, los dejo en sus respectivos lugares. Cuando les pido material y no lo traen completo, les pido que se junten de tal manera que se forme un paquete completo del material requerido (OG, p. 9).

Para fomentar la participación, los docentes utilizaban la motivación intrínseca que se generaba por que la actividad fuera interesante o lúdica: "Les dije que íbamos a jugar, que formaran equipos de tres" (HO, p. 19) o utilizaban premios como puntos para la calificación, puntos canjeables por útiles escolares, dulces, etc. Un ejemplo de premios es el de la profesora LB, que escribe: "Al final cambian sus puntos: una pluma de gel de colores, 
1000; una pluma normal, 800; un lápiz de los buenos, 100; un dibujo tamaño carta, 50” (p. 17).

En las bitácoras hay 15 evidencias claras de que algunos docentes planeaban la actividad colaborativa, entre ellas que los alumnos habían llevado materiales que les había encargado la profesora en la clase previa: juegos de geometría, fomy, papel, palitos, hojas de colores, etc., o que ella llevaba dados, premios, hojas impresas, cartulinas y presentaciones: "Los ponía en equipos de no más de 3 integrantes y les entregaba hojas de colores con los ejercicios; cada hoja (2 hojas) contenia 9 problemas variados" (SN, p. 3).

La consigna principal que los docentes daban a los alumnos para el trabajo colaborativo era que se reunieran en equipos e hicieran una actividad; por lo general no asignaban funciones y no pedían cuenta de la aportación de cada miembro del grupo. Solamente en el caso de que asignaran a un monitor había una actividad específica para uno de los integrantes. Al parecer, los profesores creen que la mera grupalidad y una actividad a realizar son suficientes para promover el aprendizaje.

En ninguna de las bitácoras apareció información acerca de que se pidiera a los estudiantes que reflexionaran sobre su desempeño en el grupo ni de que evaluaran lo que habían generado como grupo. El énfasis de los docentes estuvo en mantenerlos en la actividad y en evaluar los resultados en la clase colectiva; es decir, hubo poca atención al proceso de aprendizaje en equipos colaborativos.

Como se aprecia, existen variadas maneras de implementar las modalidades del trabajo colaborativo en el aula, en que se busca que cada integrante enriquezca su conocimiento y comprensión a partir de las aportaciones y razonamientos de sus compañeros, ya sea en binas, en equipos o en equipos con un tutor. También hay evidencias de que muchas de estas actividades fueron planeadas; sin embargo, no se enseñó a los estudiantes a trabajar en equipo y en ningún momento se les pidió que reflexionaran sobre el proceso de aprendizaje. 


\section{Contenidos y propósitos para esta modalidad de trabajo.}

Los contenidos que se utilizaban para el trabajo colaborativo eran prácticamente los mismos que en cualquier otra modalidad de enseñanza: operaciones con enteros, fracciones y decimales; geometría, particularmente medición de figuras; medidas de longitud, superficie, tiempo y capacidad; ecuaciones, interpretación de información y representación gráfica. Es decir, lo que señale el currículo oficial: sentido numérico y pensamiento algebraico, forma, espacio y medida y manejo de la información. La diferencia estaba en la manera de abordar tales contenidos.

Por lo general, cuando los profesores enseñan al grupo completo, les explican conceptos y les muestran procedimientos algorítmicos anotándolos paso a paso en el pizarrón. Cuando les piden que hagan trabajo individual, usualmente es para que practiquen algún procedimiento o resuelvan problemas sencillos. Para el trabajo colaborativo solían elegir, principalmente, actividades de resolución de problemas, descubrimiento de patrones, comprobación, prueba de hipótesis y extracción de conclusiones a partir de información dada.

El trabajo colaborativo en más de la mitad de las ocasiones se utilizó para resolver problemas como los siguientes: "Después de alcanzar una altura de 3795 metros sobre el nivel del mar, un cohete suelta una de sus turbinas y esta cae en el océano a una profundidad de -792 metros. ¿Qué distancia recorre la turbina? ¿Por qué se emplean números negativos para representar la distancia que se sumerge la turbina en el océano?" (CF, p. 22), o "Una revista de ciencia publicó que uno de los primeros satélites que existieron tardaba 95.57 minutos en dar una vuelta a la Tierra. De acuerdo con esta información: a) ¿Cuántos minutos tardaba el satélite para dar 9.5 vueltas a la Tierra?; b) ¿Cuántos minutos tardaba para dar 100 vueltas?; c) ¿Cuántos días tardaba en dar 100 vueltas? y d) ¿Cuántas horas tardaba en dar 100 vueltas?" (BL, p. 43). 
El descubrimiento es otro de los propósitos de los docentes. RS pide a los alumnos que "construyan figuras planas regulares: triángulo equilátero, cuadrado, pentágono y hexágono, para que enseguida encuentren similitudes y diferencias (p. 6). O que dupliquen, tripliquen y reduzcan a la mitad la medida de los lados de un polígono regular para que descubran a) ¿Qué sucede con el perímetro?; b) ¿Qué sucede con el apotema? y c) ¿Qué sucede con el área?

Esta modalidad de trabajo se presta, además, para que los estudiantes hagan comprobaciones: "En grupos van a la cancha de basquetbol y miden los círculos que están pintados en el piso; después miden otros círculos de diferentes tamaños y comprueban el valor de pi" (MR, p. 13). Otro profesor les pidió que comprobaran el teorema de Pitágoras y, otro docente, la fórmula para sacar el área del cuadrado, rectángulo y triángulo calculando las unidades cuadradas que tenían.

Los docentes también utilizan el trabajo colaborativo para que los alumnos aprendan a formular y probar hipótesis. Les da la consigna a los equipos de que cada integrante haga una predicción acerca del número de veces que saldrá una cara determinada de un dado al lanzarlo 60 veces; les solicita hacer una tabla, ver si su hipótesis se confirmó y tratar de explicar la razón (HO, p. 19).

El análisis de información es abordado, igualmente, a través del trabajo colaborativo para que los estudiantes aprendan a extraer conclusiones de información que se les presenta. El profesor BP da a los equipos la siguiente instrucción: "Analicen la siguiente gráfica de barras que muestra los resultados de una encuesta a un grupo de alumnos respecto a su deporte favorito. Posteriormente, contesten las preguntas" (p. 24). Se espera que los alumnos aprendan a analizar la información y lleguen a conclusiones a partir de ella.

Aunque los contenidos del trabajo colaborativo y de otras modalidades de actividad en el aula son los mismos, el propósito difiere marcadamente. 
En la exposición explican conceptos y modelan procedimientos, mientras que, en el trabajo grupal, el objetivo es resolver un problema, descubrir, probar hipótesis o extraer conclusiones.

\section{La valoración del docente.}

En las bitácoras revisadas, los profesores mencionan en 165 ocasiones algún comentario evaluativo acerca del trabajo colaborativo: 79 menciones positivas, 23 negativas, 32 ventajas y 31 dificultades de esta modalidad. Los docentes evalúan de manera muy distinta, dependiendo de la experiencia particular que han tenido en el aula al implementarla.

Entre las 79 menciones positivas, los profesores hablan de que mediante la colaboración aumentan la motivación y el entusiasmo, que mejora la disciplina, que mejora la comprensión de los estudiantes que van más rezagados debido al apoyo de los otros, que se muestran más activos e interesados y se incrementa el entusiasmo por aprender.

Una de las principales ventajas que informan los docentes es la motivación y el interés: "Los alumnos estuvieron motivados en la solución de estos cuadrados y me solicitaron que les dejara más para hacer en su casa" (VH, p. 1). "La verdad, me sentía bien de ver el interés que tenían por la actividad" (SF, p. 4). "Realmente me han sorprendido con el entusiasmo que muchos jóvenes han puesto para diseñar sus proyectos" (AG, p. 28).

También señalan el gusto de los estudiantes por la actividad: "El 100\% dijo que les gustó mucho trabajar en el equipo y que les sirvió para practicar las ecuaciones" (VF, p.2), y la participación: "Estuvieron muy participativos" (CF, p. 22). "He percibido que los alumnos son participativos, casi a todos les gusta preguntar, muestran disposición al momento de realizar las actividades" (GL, p. 40).

Asimismo, destacan la importancia del intercambio de opiniones que enriquecen el trabajo, pues frecuentemente encuentran diversas maneras de solucionar los problemas y en el debate se dan aprendizajes importantes: " $\mathrm{La}$ 
verdad es que me dejaron sorprendido por su manera de trabajar, con buen ritmo y con una gran cantidad de ideas diferentes" (PL, p. 69).

Una de las ventajas que ven es que hay estudiantes con más conocimiento que ayudan a otros a que aprendan: "El trabajo en equipo que ellos mismos hacen es excelente, hay niños monitores y me ayudan demasiado en facilitarme el trabajo en clase" (RM, p. 38). "Con los equipos, descargo responsabilidades en los alumnos más capaces, y estos se encargan de orientar a sus compañeros, y créanme si lo hacen mejor que yo con todo el grupo. Sí funciona, créanlo" (RL, p. 55).

Una profesora manifiesta así su entusiasmo por el trabajo colaborativo: "Por primera vez, en casi 16 años, me siento completamente satisfecha del resultado que pude observar en los alumnos" (CF, p. 22). Todos los docentes incluyeron en sus bitácoras una gran cantidad de fotografías del trabajo colaborativo, sobre todo cuando utilizaban material concreto o cuando los alumnos construían figuras, elaboraban gráficos o hacían exposiciones llamativas.

Pero no todo fueron evaluaciones positivas, ya que varios profesores se mostraron desanimados al poner en práctica el trabajo colaborativo en sus aulas, como se puede notar en los siguientes extractos de sus bitácoras:

Muchos de los chicos han manifestado de diversas formas un pésimo desarrollo del aprendizaje colaborativo pues, si se les pide que socialicen sin una rigurosa supervisión, comienzan por divagar y acaban olvidando por completo la meta por la que están trabajando (RV, p. 8).

Otros docentes mencionan que:

Esta forma de trabajo trae como consecuencia el liderazgo de algunos estudiantes y, a su vez, la desfachatez de otros, que como resultado de la cercanía no trabajan colaborativamente, sino que en ocasiones solo esperan a que uno o dos de ellos hagan todo el trabajo (RS, p. 26).

En $1^{\circ}$ A se sientan por afinidad, no cambian de equipo, ahi los alumnos se han atenido al que sabe o bien se sientan con los que trabajan. El trabajo 
por equipo se vuelve un problema porque dejan solo al que puede resolverlo y el que trabaja termina no haciéndolo, duran más tiempo en resolverlo (SD, p. 31).

Según yo, los chicos se están haciendo responsables de su propio proceso, pero, sorpresa, se están haciendo responsables de nada, porque nada están aprendiendo, porque siguen resolviendo sobre la marcha, repitiendo modelos para después abandonarlos en algún rincón de la memoria, si acaso, pero ni se da la transferencia, ni tampoco están siendo capaces de desarrollar competencias minimas que den respuesta a sus necesidades cognitivas, tanto inmediatas como mediatas (RV, p. 24).

Una posible explicación de las dificultades que experimentaron los profesores al poner en práctica el trabajo colaborativo es que no fomentaron adecuadamente la discusión entre los integrantes del grupo, ni se estableció la estructura colaborativa apropiada. Pareciera que el profesor dejó el proceso en manos de los líderes de cada uno de los grupos para que fomentaran el aprendizaje de los demás. Así que, a pesar de que el libro del docente recomienda seguir la metodología para el aprendizaje colaborativo, no todos los profesores pudieron implementarlo.

\section{Las decisiones.}

La principal queja de los profesores acerca del trabajo colaborativo fue la indisciplina. RV escribe: "Trabajaron por equipos, en donde se mostraban muy agresivos con los alumnos más rezagados" (p. 18). También hay quien señala explícitamente: "Observé que, cuando trabajan de manera individual, ellos están más tranquilos; la disciplina es mejor" (LV, p. 15).

Hubo dos ventajas principales que ven quienes valoran positivamente este tipo de trabajo: "El ambiente se tornó agradable, divertido" (CF, p. 7), y había una mejor comprensión pues, cuando uno comprendía o solucionaba un problema, apoyaba a sus compañeros; "al estar trabajando en equipos, 
más de alguno lo pudo hacer y se lo explicó al resto, de modo que se desarrollaron muchos ejercicios" (Al, p. 21).

A partir de la experiencia que tuvo cada profesor en la implementación del trabajo colaborativo, tomaron la decisión de continuar fomentándolo, debido a que consideran que así aprenden mejor los estudiantes, o disminuirlo para incrementar, en su lugar, la enseñanza directa y el trabajo individual para lograr un mayor control de la conducta de los alumnos y avanzar de manera más predecible en la presentación de los contenidos que señala el programa.

\section{Discusión}

El trabajo colaborativo que se reporta en las bitácoras de los docentes muestra que estos tratan de atender las indicaciones de la autoridad, quien prescribe que deben generar situaciones motivantes y significativas para los alumnos, que fomenten el trabajo colaborativo y la autonomía para aprender (SEP, 2011), lo cual hacen de manera muy variada y con frecuencia se obtienen resultados y valoraciones diferentes. Se encontraron evidencias de planeación de situaciones de aprendizaje en muchas de las bitácoras. Tal planeación estuvo centrada en diseñar situaciones para promover la resolución de problemas, el descubrimiento y el planteamiento de hipótesis, entre otros. Para ello preparaban materiales, ejercicios y problemas que, en ocasiones, estaban relacionados con los intereses o vivencias de los estudiantes.

Los profesores estaban centrados en que los alumnos se mantuvieran en la actividad en que elaboraran los productos solicitados, pero no siempre tuvieron éxito. El trabajo colaborativo en sí mismo no fue planeado ni fue objeto de revisión por docentes y estudiantes. Pareciera que los profesores consideran que la asignación de una tarea, proporcionar materiales y agrupar a los alumnos es suficiente para detonar la colaboración y el aprendizaje, al contrario de lo que sostienen los estudiosos del tema: "Nos referimos a una forma más sofisticada de trabajo en grupo donde, sobre todo, se promueve la interdependencia positiva entre los miembros del equipo y la necesidad de aportación de cada miembro para lograr el objetivo" (Duran, 2006, p. 7). 
Aunque en términos generales se le puede llamar trabajo colaborativo a lo que ocurre en el aula, si se entiende como "el empleo didáctico de grupos reducidos en los que los alumnos trabajan juntos para maximizar su propio aprendizaje y el de los demás" (Johnson, Johnson \& Holubec, 1999, p. 14), la implementación fue sumamente pobre dado que no había una estructura como la que propone Gillies (2014) o la que propusieron Johnson y Johnson (1990), y que se presentó previamente en este trabajo.

Convendría que, en lugar de solo agrupar a los estudiantes y proporcionarles un objetivo y un tiempo determinado para alcanzarlo, se establezca, como afirma Duran (2011), una interacción altamente estructurada que se centre más en el aprendizaje que en el logro del objetivo.

\section{Conclusiones}

Si, como refieren Pai, Sears y Maeda (2014), el trabajo en grupos pequeños ha mostrado tener un impacto en la transferencia del aprendizaje, comparado con el aprendizaje individualista, convendría que los profesores conozcan la fundamentación de este tipo de intervención educativa y las maneras específicas de plantear el objetivo del trabajo, los roles de los participantes, la estructura temporal, las formas de relación que se fomentan el aprendizaje, así como la comunicación de los resultados y la reflexión sobre el proceso de trabajo mismo y de su utilidad, para que los alumnos construyan nuevos conocimientos matemáticos.

Haber tomado un curso de aprendizaje colaborativo, al parecer, motivó a los profesores a utilizar más esta modalidad de aprendizaje en sus aulas. Sin embargo, la motivación no fue suficiente; hizo falta una implementación más parecida a lo que señalan quienes han aplicado exitosamente esta manera de promover el aprendizaje.

Plantear un problema a los estudiantes y pedirles que trabajen en grupo para resolverlo constituye un elemento del trabajo colaborativo, pero además es necesario enseñar a los alumnos cómo organizarse e identificar los roles que 
ha de jugar cada uno, el tiempo de que disponen para realizar la actividad y el producto que habrán de tener como resultado de la misma. Adicionalmente, habrá que enseñarles a planear el trabajo, a asignarse responsabilidades y a utilizar criterios para evaluar tanto el producto como el proceso de su trabajo.

Mientras los profesores no apliquen adecuadamente el aprendizaje colaborativo como método pedagógico, tendrán resultados distintos a los que indican las investigaciones que describen prácticas exitosas, y se sentirán frustrados y desmotivados a continuar con su aplicación. Por lo tanto, es necesario que, además de tomar cursos sobre el tema, existan otros mecanismos que ayuden a la implementación exitosa, como el acompañamiento de un docente más experto, el trabajo en comunidades de práctica o en colegios académicos.

\section{Referencias}

Andrews, T. M., Leonard, M. J., Colgrove, C. A., \& Kalinowski, S. T. (2011). Active learning not associated with student learning in a random sample of college biology courses. CBE Life Sciences Education, 10, 394-405. http://dx.doi.org/10.1187/cbe.11-07-0061

Bliss, C. A., \& Lawrence, B. (2009). Is the whole greater than the sum of its parts? A comparison of small group and whole class discussion board activity in online courses. Journal of Asynchronous Learning Networks, 13(4), 25-39.

Brown Wright, G. (2011). Student-centered learning in higher education. International Journal of Teaching and Learning in Higher Education, 23, 92-97.

Celikten, O., Ipekcioglu, S., Ertepinar, H., \& Geban, O. (2012). The Effect of the Conceptual Change Oriented Instruction through Cooperative Learning on 4th Grade Students' Understanding of Earth and Sky Concepts. Science Education International, 23(1), 84-96.

Duran, D. (2006). Tutoría entre iguales, la diversidad en positivo. Aula de Innovación Educativa, 15(153-154), 7-10. 
Gillies R. (2014). Cooperative Learning: Developments in Research. International Journal of Educational Psychology, 3(2):125-140.

Gillies, R. (2008). The effects of cooperative learning on junior high school students' behaviours, discourse and learning during a science-based learning activity. School Psychology International, 29, 328-347. http:// dx.doi.org/10.1177/0143034308093673

Gómez, L. F., Silas, J.C., \& Montoya, E. M. (2015). Un modelo para la enseñanza de las matemáticas en secundaria. Diálogos sobre educación, 6(10), 1-17.

Gómez, L. F., \& Silas, J. C. (2016). La comunidad virtual de práctica. Alternativa para la formación continua de profesores. CPU-e, Revista de Investigación Educativa, 22, 28-51.

Gómez, L. F., \& Silas, J. C. (2015). Las creencias epistemológicas de alumnos y profesores de $1^{\circ}$ de secundaria. Diálogos sobre educación, 5 .

Guzmán, K., \& Rojas-Drummond, S. (2012). Escritura colaborativa en alumnos de primaria: Un modo social de aprender juntos. Revista Mexicana de Investigación Educativa, 17(52), 217-245.

Hattie, J. A. C. (2009). Visible learning: A synthesis of over 800 metaanalyses relating to achievement. London: Routledge.

Hooker, D. (2011). Small Peer-Led Collaborative Learning Groups in Developmental Math Classes at a Tribal Community College. Multicultural Perspectives, 13(4), 220-226. http://dx.doi.org/10.1080/1 5210960.2011 .616841

Johnson, D., \& Johnson, R. (2000). Cooperative learning, values, and culturally plural classrooms. In. M. Leicester, C. Modgil, y S. Modgil (Eds.), Classroom issues: Practice, pedagogy and curriculum (pp. 1528). Palmer Press: London.

Johnson, D., \& Johnson, R. (2002). Learning together and alone: Overview and meta-analysis. Asia Pacific Journal of Education, 22, 95-105. http:// dx.doi.org/10.1080/0218879020220110

Johnson, D., Maruyama, G., Johnson, R., Nelson, D., \& Skon, L. (1981). Effects of cooperative, competitive, and individualistic goal structures on achievement: A meta-analysis. Psychological Bulletin, 89, 47-62. http://dx.doi.org/10.1037/0033-2909.89.1.47 
Johnson, D., Johnson, R., \& Holubec, E. (1999). El aprendizaje cooperativo en el aula. Buenos Aires: Paidós.

Marušić, M. M., \& Sliško, J. (2014). Students' Experiences in Learning Physics: Active Learning Methods and Traditional Teaching. LatinAmerican Journal Of Physics Education, 8(4), 4510-1-4510-12.

Mejía-Arauz, R., Keyser Ohrt, U., \& Correa-Chávez, M. (2013). Transformaciones culturales y generacionales en la participación colaborativa de niñas y niños de una comunidad P'urhépecha. Revista Mexicana de Investigación Educativa, 18(59), 1019-1045.

Mercer, N., \& Littleton, K. (2007). Dialogue and the development of children's thinking: A sociocultural approach. Londres: Routledge.

Miles, M. B., \& Huberman, A. M. (1994). Qualitative data analysis: An expanded sourcebook ( $2^{\mathrm{a}}$ ed.). Thousand Oaks, CA: Sage.

Millis, B. J. (2014). Using Cooperative Structures to Promote Deep Learning. Journal on Excellence In College Teaching, 25(3/4), 139-148.

Moliner, L., Flores, M., \& Duran, D. (2011). Efectos sobre la mejora de las competencias lingüísticas y la autoimagen lectora a través de un programa de tutoría entre iguales. Revista de Investigación en Educación, 9(2), 2011, 209-222.

National Council of Teachers of Mathematics. (1989). Curriculum and evaluation standards for school mathematics. Reston, VA: Author.

National Council of Teachers of Mathematics. (2000). Principles and standards for school mathematics. Reston, VA: Author.

OCCDE. (2013). oecd.org. Recuperado de: http://www.oecd.org/pisa/ keyfindings/pisa-2012-results-overview.pdf

Pai, H. H., Sears, D. A., \& Maeda, Y. (2015). Effects of small-group learning on transfer: A meta-analysis. Educational Psychology Review, 27(1), 79102. http://dx.doi.org/10.1007/s10648-014-9260-8

Rogoff, B. (2003). The cultural nature of human development. New York: Oxford University Press.

SEP (2011b). Programas de estudio 2011. Guía para el maestro. Educación Básica Secundaria. Matemáticas. México, D.F.: SEP. 
Slavich, G., \& Zimbardo, P. (2012). Transformational Teaching: Theoretical Underpinnings, Basic Principles, and Core Methods. Educational Psychology Review, 24(4), 569-608. http://dx.doi.org/10.1007/s10648012-9199-6

Slavin, R. (1996). Research on cooperative learning and achievement: What we know, what we need to know. Contemporary Educational Psychology, 21, 43-69. http://dx.doi.org/10.1006/ceps.1996.0004

Slavin, R., \& Cooper, R. (1999). Improving intergroup relations: Lessons learned from cooperative learning programs. Journal of Social Issues, 55, 647-663. http://dx.doi.org/10.1111/0022-4537.00140

Strauss, A. (1987). Qualitative analysis for social scientists. Cambridge. University Press. http://dx.doi.org/10.1017/cbo9780511557842

Valdebenito, V., \& Duran, D. (2013). La tutoría entre iguales como un potente recurso de aprendizaje entre alumnos: efectos, fluidez y comprensión lectora. Perspectiva Educacional, 52(2), 154-176. http:// dx.doi.org/10.4151/07189729-Vol.52-Iss.1-Art.141.

Vygotsky, L. S. (1979). El desarrollo de los procesos psicológicos superiores. Buenos Aires: Grijalbo.

Wertsch, J. (1988). Vigotsky y la formación social de la mente. Barcelona: Paidós.

You, L. (2014). Cooperative Learning: An Effective Approach to College English Learning. Theory \& Practice. In Language Studies, 4(9), 19481953.

Zabalza, M. (2004). Diarios de clase: Un instrumento de investigación y desarrollo profesional. Madrid: Narcea. 